\title{
Monitoramento biológico: conceitos e aplicações em saúde pública
}

\author{
Biological monitoring: \\ concepts and applications in public health
}

\author{
Fatima Pivetta 1 \\ Jorge Mesquita Huet Machado 1 \\ Ulisses César Araújo 1 \\ Maria de Fatima Ramos Moreira 1 \\ Pietro Apostoli 2
}

\footnotetext{
1 Centro de Estudos da Saúde do Trabalhador e Ecologia Humana, Escola Nacional de Saúde Pública Fundação Oswaldo Cruz. Rua Leopoldo Bulhões 1480, Rio de Janeiro, $R J$ 21041-210, Brasil.

2 Cattedra di Medicina del Lavoro, Università degli Studi di Brescia. Ple Spedali Civile, 1 -25123, Brescia, Italia.
}

\begin{abstract}
This study provides an overview of the theoretical discussion on potential uses for biological monitoring of exposure to chemical substances as related to human health, considering different concepts: definitions, uses, and limitations of internal dose and biological effect indicators and their availability for the substances to be quantified; knowledge of reference values, action levels, and limits based on health and negotiated patterns in biological monitoring interpretation and perspectives; and ethical and social problems in practice and within different preventive practices and their use in public health. Biological monitoring is the result of an exposure situation with conclusions based on scientific and consensus values, rules, and legislation. Biological monitoring as a continuous process and related to actually observed cases has helped establish technological exposure reference values and consensus levels as indicators for improving the environment and the workplace. As a step in the decision-making process in risk analysis, biological monitoring needs to be critically assessed as to its ethical aspects in light of the end use of results and values, which are references for application of this methodology.
\end{abstract}

Key words Environmental Monitoring; Environmental Health; Occupational Health

Resumo Neste trabalho, é apresentado um panorama das discussões teóricas sobre as potencialidades da utilização do monitoramento biológico (MB) na vigilância à saúde, nos casos de exposição a substâncias químicas, particularmente em ações de saúde ambiental e dos trabalhadores, considerando os seus diferentes conceitos; aplicação e limitações dos indicadores biológicos e sua disponibilidade para a quantificação das substâncias; valores de referência para interpretação dos resultados; os problemas éticos e sociais na prática do MB e sua inserção entre as diversas práticas preventivas e seu uso em saúde pública. Na prática, o MB constitui-se em um laudo de uma situação de exposição, tendo suas conclusões fundamentadas em valores científicos, de negociações internas, normas e legislações. Como um processo contínuo e relacionado a casos concretos de observação, tem sido uma prática promissora, para a melhoria constante dos ambientes de trabalho. Como uma etapa de um processo decisório nas análises de risco, o monitoramento biológico deve ser permeado pela avaliação crítica no que se refere aos aspectos éticos, a que se destinam os resultados, e aos próprios valores que referenciam a sua aplicação. Palavras-chave Monitoramento Ambiental; Saúde Ambiental; Saúde Ocupacional 


\section{Conceitos e aplicações do monitoramento biológico}

A partir da segunda metade dos anos 70 , as pesquisas sobre monitoramento biológico (MB) aumentaram de modo quase exponencial. Isto é comprovado pelo grande número de publicações nos principais bancos de dados biotoxicológicos, onde monitoramento biológico aparece como palavra-chave. Esse número passou de cerca de 150 no triênio 1977-1979 para mais de 5.200 na década de 90 .

Entre os principais fatores que provocaram esse avanço acentuado estão:

- O interesse nas atividades de prevenção, entendidas como principal meio para limitar as patologias que estão correlacionadas ao ambiente;

- A demonstrada (ou suposta) associação entre alguns parâmetros biológicos e as alterações do estado de saúde;

- O extraordinário desenvolvimento das técnicas analíticas, que permitiram individualizar e quantificar parâmetros biotoxicológicos, até há pouco tempo, inimagináveis.

$\mathrm{O} \mathrm{MB}$ teve diversas origens, ou melhor, foi concebido e praticado com diversas finalidades e, ainda hoje, é diversamente entendido. Uma clara demonstração dessa diversidade de entendimentos é evidenciada pelo elevado número de definições propostas, que, em alguns casos, são também sensivelmente diferentes (Bernard \& Lauwerys, 1986; Droz et al., 1991; Fiserova-Bergerova, 1987; Schulte et al., 1987; Zielhuiis, 1978).

Na sua acepção geral e originária, monitoring (atividade de olhar, observar, controlar qualquer coisa com um fim definido) era entendida como medida continuada de uma variável no tempo, através de uma atividade repetitiva e regular. Na área ambiental, o monitoramento foi sempre entendido como atividade preventiva, desenvolvida para evidenciar ou medir um risco.

Passando à definição de biological monitoring, alguns conceitos foram perdidos, tais como aqueles de necessidade da repetitividade e da regularidade das medidas. Isso se pode verificar analisando o que resta, talvez ainda hoje, da definição mais completa do $\mathrm{MB}$, formulada dentro do acordo UNEP (United Nations Environmental Programme)/WHO (World Health Organization)/EEC (European Economic Community), de 1984. Nessa formulação, o MB é definido como a medição e quantificação de substâncias químicas, ou de seus metabólitos, em tecidos, fluidos, secreções, excreções, ar expirado ou em quaisquer combinações, conduzidas para avaliar exposições e riscos à saúde, comparadas a uma referência apropriada (Berlin et al., 1984).

As numerosas e diversas definições que, antes e depois de 1984, foram propostas para o MB podem ser assim agrupadas:

a) Definições de origem-utilização prevalentemente higienística-industrial, nas quais o monitoramento resulta como uma avaliação da exposição (ocupacional) complementar, e não substitutiva, ao monitoramento ambiental, a fim de fornecer informações adicionais no enquadramento dos riscos; identificar indivíduos expostos a maiores-menores riscos; permitir o controle da eficiência dos sistemas preventivos; permitir a avaliação da absorção cutânea e gastrointestinal dos tóxicos, além da respiratória (ACGIH, 1989; DFG, 1991; Fiserova-Bergerova, 1987)

b) Definições de natureza biotoxicológica, reconhecendo-se a capacidade do MB de uma avaliação direta da quantidade de substância química efetivamente absorvida e, assim, do risco. Como indicador de dose interna, é o MB, consequentemente, mais bem correlacionado com a resposta e os efeitos biológicos do que com o efeito ambiental e, ainda, possibilita comprovar a exposição do indivíduo e a presença da substância no organismo, ou seu metabólito; mensurar a associação entre as substâncias químicas e riscos e avaliar mudanças que se refletem numa resposta ou efeito tóxico (Bernard \& Lauwerys, 1986; Henberg \& Aitio, 1987; Schulte, 1991; Zielhuiis, 1987). O MB assume assim uma maior "dignidade", sendo aplicável como prática autônoma em condições de fornecer informações sobre as exposições e os efeitos.

c) Definições que prevêem uma aplicação epidemiológica para o MB, ou melhor, uma utilização com grupos de sujeitos. Pode-se antever uma utilidade no estudo da distribuição de parâmetros toxicológicos a correlacionar com as suas fontes-causas ou com efeitos, comprovados ou suspeitos, sobre a saúde, podendo, assim, mensurar diretamente a espécie e a distribuição dos tóxicos na população; confrontar os indicadores biológicos com as modificações do estado de saúde (Bertazzi \& Duca, 1987; Margolin, 1988; Schulte, 1987).

Com esta última definição, podem ser recuperados alguns dos conceitos do monitoramento, entendido como prática repetitiva e regular em condições de avaliar as mudanças espaço-temporais dos indicadores. 


\section{Os indicadores biológicos de dose interna e de efeito}

O emprego dos indicadores biológicos de dose interna tem como objetivo a demonstraçãoquantificação da exposição-absorção de xenobióticos. Estes indicadores podem refletir a dose da substância no sítio de ação, a dose armazenada em um ou em vários compartimentos ou aquela recentemente absorvida (Araújo, 1995).

Os indicadores de efeito devem ser capazes de demonstrar e quantificar, em uma correlação definida ou previsível com os indicadores de dose, um efeito no órgão crítico. $\mathrm{O}$ uso desses indicadores combinado com os de dose interna permite explorar o intervalo existente entre a exposição e a doença, ainda em investigação, para a maioria dos xenobióticos. Ambos os indicadores, quando apresentam uma correlação significativa com a exposição, são denominados indicadores biológicos de exposição (Araújo, 1995; Schulte, 1989).

Os indicadores biológicos podem ser utilizados em diversas etapas e com significados diferentes. Na maior parte das situações de nosso interesse, permitem explorar-quantificar a dose biologicamente ativa e os efeitos biológicos precoces.

Não dispomos de indicadores biológicos de igual significado e valor para todos os xenobióticos. Dessa aparentemente óbvia constatação, podem-se tirar algumas considerações de natureza geral, como os assim chamados critérios de admissibilidade ao emprego de um indicador, isto é, os critérios mínimos que devem ser respeitados para a utilização de um indicador no MB.

Estes critérios podem ser assim agrupados: possibilidade de dosagem em amostras biológicas facilmente obtidas, transportáveis e conserváveis; existência de métodos analíticos suficientemente sensíveis, precisos e acurados; suficiente grau de conhecimento tóxico-cinético; conhecimento do comportamento dos indicadores utilizados em relação à exposição, considerando, em particular, a natureza, duração e eventual tempo até o término da exposição; conhecimento de todos os fatores fisiológicos e patológicos (independente da exposição), que podem condicionar os níveis biológicos do indicador; conhecimento sobre correlações entre dose-efeito e dose-resposta (Alessio et al., 1984).

Destaca-se, entretanto, o aspecto de como os conhecimentos toxicocinéticos, oportunamente definidos como os conhecimentos do que faz o organismo ao tóxico (enquanto os toxicodinâmicos são o que faz o tóxico ao organismo), como requisito essencial para trans- formar um número em um dado analítico biologicamente significativo. $\mathrm{O}$ conhecimento da toxicocinética de eliminação de uma substância, por exemplo, é muito importante na escolha do indicador. No caso de agentes químicos com meia-vida menor do que duas horas, a necessária alta freqüência de amostragem reduz a possibilidade aplicativa do $\mathrm{MB}$, bem como daqueles cuja cinética de eliminação tem uma variabilidade grande no tempo, por exemplo o cobalto urinário (Aitio \& Kallio, 1999).

Se o indicador é utilizado para o estudo de alterações do estado de saúde ou é relacionado com determinados padrões, como os níveis de ação ou de valores-limite, são necessários, então, outros conhecimentos, como aqueles sobre relações dose-resposta e dose-efeito, indispensáveis para relacionar os dados biológicos com os efeitos adversos à saúde.

Nem todos os indicadores são iguais ou têm o mesmo valor para a avaliação da exposição. Por essa razão, muito oportunamente, está-se desenvolvendo a proposta de distinguir cada indicador, com uma pontuação que evidencie, imediatamente, a sua qualidade. Talvez, hoje, seja difícil pensar numa explicação deste tipo, baseada em escala, para os indicadores, mas é uma maneira possível de identificar prontamente o potencial informativo dos indicadores biológicos mais requisitados ou utilizados.

\section{Quais substâncias ou compostos devem ser quantificados?}

A resposta à questão "Quais substâncias ou compostos devem ser quantificados?" pode aparecer deduzida: em que interessa ou para que serve quantificar ou o que é possível quantificar?

Do total de substâncias químicas existentes (cerca de cem mil), foram realizados estudos da possível toxicidade, experimental ou humana, para apenas seis a sete mil. Muito menos ainda são aquelas para as quais foram propostos métodos analíticos: 190, pelo Centers for Disease Control and Prevention (CDC), dos Estados Unidos; 100, pelo National Institute of Occupational Safety and Health (NIOSH), dos Estados Unidos; 120, pelo Deutsch Forschungsgemeinschaft (DFG), da Alemanha. Atualmente, a American Conference of Governmental Industrial Hygienists (ACGIH) e o DFG têm estabelecidos valores de limites biológicos para 37 e 43 compostos, respectivamente (ATSDR, 1989; DFG, 1991; Morgan \& Schaller, 1999; NIOSH, 1987).

Para identificar e classificar substâncias de interesse ecotoxicológico, a Environmental Pro- 
tection Agency/Agency for Toxic Substances and Disease Registry (EPA/ATSDR) propôs um método original, que compreende o estabelecimento dos critérios e métodos para o MB e determinação dos valores de referência. Essa classificação considera três fatores: a toxicidade intrínseca de cada substância, a freqüência com a qual estas podem ser encontradas no ambiente e a probabilidade de o homem estar exposto. Sobre esta base foi produzida a Priority Hazardous Substances List de 225 compostos distribuídos em grupos de prioridades (ATSDR, 1989).

Em outros países, como a Itália, a questão sobre indicadores biológicos foi abordada pela primeira vez no 41으 Congresso Nacional da Sociedade Italiana de Medicina do Trabalho, em 1978 (Abbritti et al., 1985; Brugnone et al., 1985; Foà et al., 1978).

No Brasil, em 1983, o Ministério do Trabalho editou a primeira portaria que definia indicadores biológicos de exposição para 29 agentes químicos em ambientes de trabalho. Os valores estabelecidos nessa portaria e nas subseqüentes não são baseados em estudos nacionais, mas adotados de outros países, principalmente dos Estados Unidos. Atualmente, estão estabelecidos na legislação brasileira, Quadro I da Norma Regulamentadora NR-7 (Brasil, 1999), indicadores biológicos para 26 agentes químicos.

\section{Como interpretar os resultados do monitoramento biológico: valores-guia}

Na definição de $\mathrm{MB}$, apresentado no primeiro item deste artigo, está explícito que o resultado da medição dos indicadores deve ser comparado com uma referência apropriada.

As referências com as quais se comparam os resultados do MB poderiam, talvez, ser melhor definidas como valores operativos ou guias, por permitirem uma orientação na interpretação dos resultados da análise. Esses valores deveriam fornecer com rapidez dois tipos de informação:

- A comparação entre o resultado do MB e os valores medidos em populações, para as quais se exclui uma específica e significativa exposição à substância em questão, deveria permitir a correlação com o grau de exposição-absorção. - O resultado do MB deveria orientar com relação ao aparecimento de determinados efeitos, correlacionando-os a possíveis alterações do estado de saúde, com base nos valores para os quais foi atribuído, científica ou convencionalmente, um significado.
Os valores-guia são assim reagrupáveis:

a) Valores de referência - definidos como as concentrações das substâncias químicas encontradas em pessoas não expostas, vivendo em situações similares às do grupo em estudo; são os valores considerados como normais. Em virtude das mudanças dos padrões de exposição da população em geral a diversas substâncias e, também, ao aperfeiçoamento das técnicas analíticas, esses valores vêm sendo reduzidos ao longo do tempo (Apostoli, 1999).

b) Níveis de ação - inferiores aos valores-limite (geralmente metade ou um terço dos mesmos) abaixo dos quais julga-se não ser necessário um controle biológico ou sanitário freqüente (menos de um ano). Conceitualmente, vem colocado por alguns autores em uma posição intermediária entre os valores-limite e os valores de referência, enquanto outros fazem coincidir com o valor superior do intervalo de distribuição dos próprios valores de referência.

c) Limites baseados em saúde - definidos como os valores para os quais não se manifestam efeitos adversos mensuráveis sobre a saúde. Esses valores se baseiam em avaliações científicas e derivam das relações dose-efeito e dose-resposta, sem levar em consideração problemas de ordem técnica ou econômica. São sujeitos, exclusivamente, às mudanças que se originam das novas informações toxicológicas.

d) Padrões negociados - valores-limite que resultam da incorporação-integração dos valores de natureza não científica, mas de ordem tecnológica, econômica, social ou política. São resultados de compromissos e comportam, por definição, a aceitação de uma certa quantidade de risco. Entre esses valores, encontram-se aqueles fixados pelas entidades supranacionais, governo, associações ou entidades corporativas.

Vale ressaltar que, com relação às substâncias carcinogênicas, existem muitas controvérsias sobre o estabelecimento ou não de valores-guia. Até o momento, nenhum valor pode ser considerado seguro; entretanto, alguns países, como a Alemanha e os Estados Unidos, adotaram valores para identificação individual de expostos a essas substâncias, com concepções diferentes para estabelecimentos desses valores (Morgan \& Schaller, 1999).

\section{Perspectivas para o monitoramento biológico}

Para alcançar os objetivos, anteriormente citados, é necessário identificar as linhas de desenvolvimento da pesquisa sobre MB dos tóxicos ambientais e ocupacionais. 
Aparecem como prioritárias as pesquisas que permitam aumentar o número de compostos que podem ser quantificados. Considerando que os estudos desses indicadores são sempre determinações analíticas, que devem estabelecer a acurácia, a sensibilidade e a especificidade (agindo sobre seus fatores pré-analíticos e analíticos), o conhecimento das causas não analíticas de variabilidade e da toxicocinética da substância no organismo humano também é importante.

Os progressos nesses campos, além de possibilitarem a ampliação do MB para um número maior de compostos, têm também criado as bases para a determinação de valores de referência adequados.

Também importante são os estudos para a identificação de novos indicadores de interação entre xenobióticos e diversos constituintes biológicos que permitam reconhecer alterações moleculares mínimas. Aparece como promissora a utilização de adutos de macromoléculas, tais como adutos de DNA. Conceitualmente, os adutos de macromoléculas estão próximos de se tornar indicadores ideais para a avaliação de risco, pois podem fornecer uma medida integrada da exposição, representam efeitos precoces e geralmente reversíveis, além de, provavelmente, refletirem a susceptibilidade individual a agentes etiológicos. Na prática, ainda são de difícil aplicação, já que requerem métodos analíticos complexos e estão limitados a compostos, ou a seus metabólitos, que formam ligações covalentes com macromoléculas (Mutti, 1999).

Ainda que controversos do ponto de vista ético, são importantes os estudos e as aplicações já disponíveis dos indicadores de efeito imunitário, mutagênico, carcinogênico e teratogênico, pelo seu valor preditivo. Com relação aos indicadores de efeito carcinogênico, há pouca validação qualitativa para efeitos a longo prazo para aberrações cromossômicas. Os indicadores não são específicos em relação aos agentes químicos e não estão validados para a avaliação quantitativa da seqüência: exposição, absorção, dose interna e dose-alvo. Os métodos analíticos não estão bem estabelecidos e a garantia de qualidade externa, geralmente, não disponível (Aitio \& Kallio, 1999; BrandtRauf, 1988; CEC, 1988; Stein \& Hatch, 1987).

Muitos compostos químicos apresentam diferentes espécies com toxicidade e metabolismo também diferenciados, o que afeta a concentração. Portanto, para entender e avaliar os mecanismos de interação entre os elementos químicos e seus alvos biológicos, a identificação e a quantificação desses elementos, em muitos casos, não são suficientes. Há necessidade do conhecimento das formas químicas em que estes se encontram nesses meios, através dos estudos de especiação química. O mercúrio, por exemplo, pode existir em três estados de oxidação, que diferem consideravelmente quanto à toxicidade (Apostoli, 1998; Das et al., 1996; Lobinski, 1997).

No campo da pesquisa biotoxicológica, ao contrário da ambiental, a especiação química ainda representa um desafio analítico em muitos aspectos metodológicos e éticos. Isto se deve a fatores tais como a reconhecida complexidade dos materiais biológicos, os níveis de concentração das substâncias químicas, em geral, muito baixos, a necessidade do entendimento do papel de cada forma específica nos processos fisiológicos e patológicos, a disponibilidade de métodos analíticos que atendam aos requisitos de qualidade aceitáveis, a praticabilidade e os aspectos éticos da amostragem (Apostoli, 1998; Mutti, 1999).

Também importante é o estudo contínuo de indicadores mais específicos e sensíveis para aquelas substâncias de impacto comprovado na saúde pública, mesmo com indicadores de dose e/ou de efeito já estabelecidos.

A aplicabilidade do MB nas pesquisas epidemiológicas supera os limites do uso de indicadores ambientais de exposição, os quais estimam apenas pontualmente os níveis de poluição. Entretanto, essa aplicabilidade ainda é restrita a poucas substâncias em virtude da nãodisponibilidade de indicadores representativos para estudos prospectivos. A maioria dos compostos tem tempo de meia-vida curto, o que dificulta a sua aplicação, pois requerem amostragens freqüentes. Atualmente, dispõe-se de indicadores aplicáveis em estudos epidemiológicos apenas para chumbo, cádmio, mercúrio e monóxido de carbono. Outro limitante para os estudos epidemiológicos é a dificuldade de acompanhamento dos sujeitos expostos, em particular, os trabalhadores, que deixam as fábricas, e, mesmo quando esse acompanhamento é factível, constata-se a perda deste para o estudo da exposição (Mutti, 1999; Vanio, 1999).

Também é um fator limitante importante para esses estudos o conhecimento sobre a toxicocinética e toxicodinâmica para um número reduzido de substâncias, porque estes dois processos controlam o desenvolvimento temporal dos efeitos, a partir da absorção de um agente químico pelo organismo (Smith, 1987). 


\section{Os problemas éticos e sociais na prática do monitoramento biológico}

As questões éticas e sociais envolvidas na prática do MB foram levantadas, inicialmente, nos Estados Unidos. Estas envolviam aspectos psicológicos, como aqueles ligados ao uso de práticas invasivas, por exemplo, a coleta de sangue, e a sensação de que um tóxico, tido como uma coisa externa ao corpo humano, tem sua presença demonstrada no próprio organismo pelo MB.

Por sua vez, os trabalhadores e seus representantes são levados a suspeitar que o MB pode ser usado para identificar indivíduos, que seguem práticas de trabalho ou higiênicas pessoais diferentes, não aceitáveis nas suas atividades, ou, ainda, que os indicadores biológicos possam demonstrar problemas de escasso rendimento no trabalho.

Os problemas relacionados à prática do $\mathrm{MB}$, cujos resultados poderiam ser usados para selecionar e excluir grupos particulares de trabalhadores (por raça, ordem genética, problemas sanitários etc.), criaram maior resistência e hostilidade à sua aplicação.

A introdução de indicadores, como os marcadores genéticos, abre um campo de discussão em relação à seleção genética de trabalhadores, tornando complexa a avaliação da legitimidade de determinadas práticas de diagnóstico preditivo (Introna, 1992).

Na realidade, o risco do uso impróprio do MB é muito mais amplo e desvirtuado. Uma das questões diz respeito à necessidade de recorrer ao seguro privado (nos Estados Unidos é quase norma), cujas possíveis exigências de testes que evidenciem predisposição, por exemplo, a doenças metabólicas ou tumores levariam, com base nos resultados obtidos, à recusa em firmar o contrato de seguro ou à solicitação de aumento considerável do mesmo a quem resultasse predisposto à determinada patologia.

Um outro emprego enganoso seria aquele voltado a identificar sujeitos particularmente resistentes aos tóxicos, para destiná-los a atividades em ambientes de trabalho perigosos, o que força a adaptação do homem ao trabalho, e não o contrário.

Os empregadores também manifestam hostilidade ao emprego do MB. As principais resistências dizem respeito ao fato de os indicadores biológicos demonstrarem uma exposiçãoabsorção de um tóxico, sem que se possa, simultaneamente, demonstrar com exatidão a ausência de qualquer efeito, e impõe a necessidade de afastamento do trabalhador sob responsabilidade da empresa. Além disso, provo- ca o temor de que, em litígio médico-legal, o MB demonstre que os trabalhadores, de algum modo, absorveram o tóxico. Em outras palavras, o MB comprovaria a hipótese ou levantaria problemas, mesmo onde não fossem ainda demonstráveis.

Os aspectos que dizem respeito às relações com o trabalho devem ser considerados de forma enfática, em países com instrumentos contratuais e de tutelas sociais mais frágeis e diferenciadas, como o Brasil. Nesses países, onde a estrutura legal e a organização dos serviços de vigilância da saúde e de assistência social são bastante precários, os níveis de educação baixos e de desemprego altos, criam-se condições para exclusão por qualquer alteração identificada nos exames. Essa situação leva a um temor que gera resistência para implantação de programas de $\mathrm{MB}$, propiciando a cultura de aceitação de riscos, o que distancia a perspectiva da influência dos trabalhadores e sindicatos nas negociações de padrões de exposição.

Por outro lado, a realização de diagnóstico de doenças a partir do MB como parâmetro único de reconhecimento de doenças, sobrevaloriza os indicadores e relega, erroneamente, os sintomas clínicos. Tais procedimentos, por sua vez, têm base na legislação, que é por natureza defasada em relação ao conhecimento científico, fazendo com que ocorram conclusões dissociadas da condição de saúde e uma exclusão artificial de casos. Essa situação é fonte de conflitos ético-profissionais e institucionais.

\section{Como colocar o monitoramento biológico entre as diversas práticas preventivas}

Alguns autores americanos da escola higienística-industrial afirmam que o MB é uma prática não necessária, ou melhor, uma prática à qual se recorre quando outras falham. De fato, se estas fossem garantidas, com intervenções preventivas (controláveis com o monitoramento ambiental), concentrações de contaminantes ambientais próximas a zero ou sua absorção em quantidade imensurável, não existiriam os pressupostos para conduzir ao MB. Por isso, o MB, não ao acaso, está entre as práticas de prevenção secundária, com o objetivo de reduzir a absorção dos tóxicos e a natureza dos seus efeitos. Porém, concentrações ou risco zero são abstrações, ao menos na quase totalidade das atuais realidades, ocupacionais e ambientais (EPA, 1976).

Assim, o MB encontra pressupostos e justificativas no âmbito higienistíco-industrial, co- 
mo atividade complementar, subordinada ao monitoramento ambiental, partindo da convicção de que este último seja um sistema mais confiável para a demonstração dos riscos ocupacionais. Isso pode, em parte, ser aceito se for limitada à atual realidade e aos compostos para os quais existem poucos estudos biotoxicológicos. Entretanto, freqüentemente, os critérios e métodos do monitoramento ambiental não são imunes às críticas, e não podem servir, por definição, para cada situação, de parâmetro absoluto para avaliar outras variáveis.

O monitoramento ambiental apresenta algumas limitações que reduzem sua capacidade de avaliar adequadamente a exposição e o risco à saúde. Entre elas, destacam-se:

- avaliação da exposição apenas pela via respiratória, enquanto, para muitas substâncias, as principais vias de absorção são a cutânea e/ ou o trato gastrointestinal;

- as constantes variações da concentração de um contaminante no ar do ambiente exigem uma freqüência de amostragem de difícil execução;

- diferenças no tamanho das partículas, na carga de trabalho, o que altera a taxa de ventilação pulmonar, e na eficácia e freqüência de utilização dos equipamentos de proteção individual podem alterar bastante a dose absorvida entre os trabalhadores expostos a uma mesma concentração da substância no ar.

As relações entre a concentração no ar, a dose interna e o efeito podem variar muito, se os parâmetros citados não forem controlados. Assim, a estimativa da exposição não fornece informações suficientes para avaliar o risco à saúde, um dos objetivos do monitoramento ambiental (Araújo, 1995).

Trata-se, pois, de reconsiderar as relações entre os dois tipos de monitoramento, partindo do princípio de que ambos devem ter validade na prática atual, mesmo com um grau de comprometimento como medida preventiva, seguramente maior para o biológico.

O MB integra todas as vias de exposição/ absorção e incorpora as demais variáveis numa mesma medida, dose interna, própria de cada indivíduo. Por intermédio dos indicadores de dose e, ainda melhor, daqueles de efeito, demonstra a existência de uma exposição individualizada real, e não mais estimada, ou alteração biológica reversível em sítios-alvo, avaliando melhor o risco. Todavia, enquanto outras práticas preventivas e a vigilância sanitária são dedicadas a demonstrar alterações de sistemas ou órgãos que portam, ou portarão, a deterioração funcional, a patologia ou a falência dos mesmos, o MB demonstra a presença dos tóxicos, seus metabólitos ou alterações precoces por eles induzidas, e se presta, pois, a fornecer informações para intervenções, antes que as alterações se transformem em efeitos adversos irreversíveis.

Trata-se de um salto conceitual em relação à avaliação de risco conduzida com os instrumentos do monitoramento ambiental, uma vez que se avaliam doses e eventuais efeitos, considerando as características do organismo de coletar, de maneira integrada, os tóxicos (fase de exposição-absorção), de tratá-los (fase toxicocinética) e de ser influenciado por eles (fase toxicodinâmica).

O MB passa, assim, da prática subalterna à natureza autônoma, sistema de alarme precoce dos riscos ambientais, sendo capaz de quantificar doses e de demonstrar alterações bioquímicas ou funcionais precoces e, previsivelmente, reversíveis.

\section{O monitoramento biológico como prática em saúde pública}

O MB é uma atividade intrínseca à vigilância em saúde do trabalhador e ambiental, que, por sua vez, tem como um de seus pressupostos a interdisciplinaridade, em virtude da natureza múltipla e ecológica de seus objetos (Machado, 1997).

Em um modelo de vigilância em saúde ou de promoção à saúde, o MB se situa na interface entre os condicionantes técnicos e sociais, os perfis epidemiológicos e de situações de saúde, fazendo parte da conexão entre o biológico e o ambiente no processo de saúde-doença, construindo a ligação entre as avaliações ambientais e de processo de trabalho com as análises clínicas e laboratoriais.

O MB em si é multidisciplinar e agrega diversas áreas do conhecimento, como a química, a biologia molecular, a bioquímica, a toxicologia e a fisiologia, entre outras.

Sua aplicação se articula e depende, intrinsecamente, de etapas que vão desde o estabelecimento de indicadores de exposição e/ou de efeitos à definição de grupos de risco, elaboração de protocolos para controle de amostras, definição de estratégias de coleta, definição de valores de referência (normas, por exemplo) e a preparação do laboratório quanto a metodologias analíticas e sistema de qualidade.

Pode ser considerada uma atividade estratégica pela sua contribuição na legitimação da área de saúde do trabalhador, pois a evidência decorrente de um resultado alterado em um MB estabelece uma relação direta de um dado 
individual, âmbito incontestável do setor saúde, com o ambiente de trabalho, fazendo a ligação das observações no indivíduo com o coletivo do trabalho.

A perspectiva do uso preventivo dos resultados do MB e da correlação com dados clínicos e epidemiológicos também colaboram nesse processo de legitimação da saúde pública no campo das relações de trabalho.

No contexto do embasamento teórico, necessário nas negociações entre trabalhadores, empresas e governo, que, constantemente, necessita de parâmetros quantitativos, a facilidade de estabelecermos correlações entre o MB e as avaliações ambientais, e mesmo a maior precisão do primeiro para definição da exposição, permitem ao MB enquadrar-se perfeitamente e, portanto, legitimar avaliações qualitativas, constituindo-se em indicadores de acompanhamento de mudanças no ambiente e processo de trabalho.

O MB é de fundamental importância na definição e redefinição de grupos homogêneos de risco, em um processo de aproximação sucessiva, em que as diferenças internas a esses grupos são levadas em consideração e redefinem um novo recorte das áreas ou situações de risco (Machado et al., 1992).

$\mathrm{O}$ MB tem como resultado um valor que corresponde à exposição individual. Esse viés da área da saúde compreende a visão de particularização dos indivíduos, podendo, ao agrupálos, construir uma visão coletiva da exposição. Essa individualização da exposição, em países de economia periférica como Brasil, tem uma particular importância devido aos processos de trabalho e tecnologias sujas, na maioria dos casos, que levam a diferentes condições de trabalho num mesmo setor de atividade produtiva.

Os resultados do MB permitem identificar grupos de risco, mesmo em populações com níveis de concentração em faixas consideradas de normalidade, ou seja, a análise de dados de grupo permite a identificação e/ou diferenciação da natureza da exposição (ocupacional ou ambiental), em faixas de concentração consideradas normal. Isso é muito importante, pois estudos de dose/resposta, cada vez mais, demonstram que ocorrem efeitos em exposições a baixas doses, como as resultantes de exposição ambiental. Em grupos de referência no interior de indústrias, como aqueles de atividades administrativas, observam-se valores dentro da faixa de normalidade, porém acima dos valores encontrados em grupos pertencentes à população em geral.

A incorporação do MB nas ações de vigilância em saúde ambiental e dos trabalhadores é ainda incipiente, embora promissora pelo seu potencial de integração técnico-científico entre distintos contextos de análise das relações saúde-ambiente. Também fortalece as ações nesses campos, uma vez que age como fator legitimador destas, considerando-se o cenário favorável da discussão em torno de condições ambientais e de mudanças tecnológicas, em que um parâmetro quantitativo tem uma contribuição importante, particularmente na pactuação sócio-técnica entre os diferentes segmentos da sociedade envolvidos.

Decorre daí, também, a sua facilidade de incorporação a estudos epidemiológicos, que potencializam a legitimação institucional do setor saúde perante as empresas e os órgãos do trabalho, meio ambiente e previdência social.

\section{Considerações finais}

Na prática, o MB, além de ter um componente individual, constitui-se em um laudo de uma situação de exposição, tendo suas conclusões fundamentadas em valores científicos, de negociações internas, normas e legislações. Como um processo contínuo e relacionado a casos concretos de observação, tem sido uma prática promissora, apontando para valores de referência tecnológicos de exposição e índices negociados, como indicadores de melhoria constante dos ambientes e locais de trabalho. Essa realidade está sendo construída, ainda que lentamente, com base em dados de exposição e valores de referência, trabalhos de pesquisa, estudos epidemiológicos e ações de vigilância.

$\mathrm{O} \mathrm{MB}$, como uma etapa de um processo decisório nas análises de risco, deve ser permeado pela avaliação crítica no que se refere aos aspectos éticos a que se destinam os resultados e aos próprios valores que referenciam a sua aplicação. 


\section{Agradecimentos}

A elaboração deste trabalho teve o apoio financeiro da Fundação de Amparo à Pesquisa do Rio de Janeiro (FAPERJ), no âmbito do convênio técnico-científico com a Università degli Studi di Brescia, Cattedra de Medicina del Lavoro, Itália (Processo no E-26/170.073/99).

\section{Referências}

ABBRITTI, G.; ALESSIO, L.; BISCALDI, G. P.; CAVALLARI, A.; FRANCO, G.; ROLAMANI, T. \& MUTTI, A., 1985. Indicatori biologici nell'esposizione professionale a metalli. In: 48o Congresso Nazionale della Società Italiana di Medicina del Lavoro e Igiene Industriale, Atti, pp. 63-130. Pavia: Monduzzi.

ACGIH (American Conference of Governmental Industrial Hygienists), 1989. Documentation of the Biological Exposure Indices. Cincinnati: ACGIH.

AITIO, A. \& KALLIO, A., 1999. Exposure and effect monitoring: A critical appraisal of their practical application. Toxicology Letters, 108:137-147.

ALESSIO, L.; BURATTI, M.; BERTELLI, G. \& DELL'ORTO, A., 1984. Significato del monitoraggio biologico. In: Monitoraggio Biologico Negli Ambienti di Lavoro (L. Alessio \& P. A. Bertazzi, ed.), pp. 23-56, Milano: F. Angeli.

APOSTOLI, P., 1998. La speciazione dei metalli in medicina del lavoro. Medicina del Lavoro, 89:3-16.

APOSTOLI, P., 1999. Application of reference values in occupational health. International Archives of Occupational and Environmental Health, 72:195204.

ARAÚJO, U. C., 1995. Avaliação da Exposição ao Chumbo em Ambientes de Trabalho: Aplicação de Parâmetros Ambientais e Biológicos. Dissertação de Mestrado, Rio de Janeiro: Escola Nacional de Saúde Pública, Fundação Oswaldo Cruz.

ATSDR (Agency for Toxic Substances and Disease Registry), 1989. Decision guide for identifying substance specific data needs related to toxicological profiles. Federal Register, 54:174.

BERLIN, A.; YODAIKEN, R. E. \& HENMAN, B. A., 1984. Assessment of Toxic Agents at the Workplace. Roles of Ambient and Biological Monitoring. Boston: Hague Niijhoft.

BERNARD, A. \& LAUWERYS, R., 1986. Present status and trends in biological monitoring of exposure to industrial chemicals. Journal of Occupational Medicine, 28:558-562.

BERTAZZI, P. A. \& DUCA, P. G., 1987. The use of biological indicators in the surveillance of groups of individual. In: Occupational and Environmental Hazards: Cellular and Biochemical Indices for Monitoring Toxicity (V. Foà, E. A. Hemmett, M. Marono \& A. Colombi, ed.), pp. 43-47, New York: John Wiley \& Sons.

BRANDT-RAUF, R. W., 1988. New markers for monitoring occupational cancer. Journal of Occupational Medicine, 30:399-404.
BRASIL, 1999. Normas Regulamentadoras de Segurança e Saúde do Trabalhador. NR-7. 25 Janeiro 2000 <http://www.mtb.gov.br/legi/nrs/nr07b.htm>.

BRUGNONE, F.; COLOMBI, A.; DE ROSA, E.; DE ZONI, R.; FRANCHI, I. \& VALENTA, T., 1985. Indicatori biologici nel monitoraggio a solventi industriali anestetici e gas tossici. In: 48o Congresso Nazionale della Società Italiana di Medicina del Lavoro e Igiene Industriale, Atti, pp. 375-472. Pavia: Monduzzi.

CEC (Commission of European Communities), 1988. Indicators for Assessing Exposure and Biological Effects of Genotoxic Chemicals. Brussels: Office for Official Publications.

DAS, A. K.; CHAKRABORTY, R.; CERVERA, M. L. \& GUARDIA, M., 1996. Metal speciation in biological fluids - A review. Mikrochimica Acta, 122:209246.

DFG (Deutsche Forschungsgemeinschaft), 1991. Maximum Concentrations at the Workplace and Biological Tolerance Values for Working Materials. Commission for the Investigation of Health Hazards of Chemical Compounds in the Work Area, Report 33. Weinheim: VCH Verlog.

DROZ, P. O.; BERODE, M. \&WU, M. M., 1991. Evaluation of concomitant biological and air monitoring results. Applied Occupational and Environmental Hygiene, 8:465-472.

EPA (Environmental Protection Agency), 1976. Interim procedures and guidelines for health risk and economic impact assessment of suspected carcinogens. Federal Register, 41:21402-21415.

FISEROVA-BERGEROVA, V., 1987. Development of biological exposure indices (BEIs) and their implementation. Applied Occupational and Environmental Hygiene, 2:87-92.

FOÀ, V.; ALESSIO, L.; CHIESURA, P.; FRANCHI, I.; MUTTI, A. \& ABBRITTI, G., 1978. Controllo sanitario e monitoraggio biologico per soggetti professionalmente esposti. In: 41o Congresso della Società Italiana di Medicina del Lavoro e Igiene Industriale, Atti, pp. 175-186. S. Margherita Ligure: Monduzzi.

HERNBERG, S. \& AITO, A., 1987. Validation of biological monitoring: An introduction. In: Occupational and Environmental Hazards: Cellular and Biochemical Indices for Monitoring Toxicity (V. Foà, E. A. Emmett, M., Maroni \& A. Colombi, ed.), pp. 41-49, New York: John Wiley \& Sons.

INTRONA, F., 1992. Screening genetico e giudizio di idoneità al lavoro. Difesa Sociale, 3:29-41. 
LOBINSKI, R., 1997. Elemental speciation and coupled techniques. Applied Spectroscopy, 5:260A278A.

MACHADO, J. M. H., 1997. Processo de vigilância em saúde do trabalhador. Cadernos de Saúde Pública 13(Sup. 2):33-46.

MACHADO, J. H.; BARCELLOS, C.; LIMA, L. \& PIVETTA, F., 1992. Arsenic exposure in the working environment of a zinc smelting pant; homogeneous group analysis. In: International Seminar Arsenic in the Environment and its Incidence on Health, Proceedings, pp. 153-158, Santiago de Chile: Universidad de Chile.

MARGOLIN, B. H., 1988. Statistical aspects of using biological markers. Statistical Science, 3:351-357.

MORGAN, M. S. \& SCHALLER, K.-H., 1999. An analysis of criteria for biological limit values developed in Germany and the United States. International Archives of Occupational and Environmental Health, 72:195-204.

MUTTI, A., 1999. Biological monitoring in occupational and environmental toxicology. Toxicology Letters, 108:77-89.

NIOSH (National Institute for Occupational Safety and Health), 1987. Registry of Toxic Effects of Chemical Substances, 1985-1986. NIOSH Publications 87-114. Cincinnati: NIOSH.

SCHULTE, P. A., 1987. Methodologic issues in the use of biologic markers in epidemiological research. American Journal of Epidemiology, 126:1006-1016.
SCHULTE, P. A., 1989. A conceptual framework for the validation and use of biologic markers. Environmental Research, 48:129-144.

SCHULTE, P. A., 1991. Contribution of biological markers to occupational health. American Journal of Industrial Medicine, 20:435-446.

SCHULTE, P. A.; HARPELIN, W. \& HERRICK, M., 1987. The current focus of biological monitoring. In: Occupational and Environmental Hazards: Cellular and Biochemical Indices for Monitoring Toxicity, Toxicity (V. Foà, E. A. Emmett, M., Maroni \& A. Colombi, ed.), pp. 50-61, New York: John Wiley \& Sons.

SMITH, T. J., 1987. Exposure assessment for occupational epidemiology. American Journal of Indus trial Medicine, 12:249-268.

STEIN, Z. \& HATCH, M., 1987. Biological markers in reproductive epidemiology: Prospects and precautions. Environmental Health Perspectives, 74: 67-75.

VANIO, H., 1999. Biomarkers in the identification of risks, especially with regard to susceptible persons and subgroups. Scandinavian Journal of Work, Environmental and Health, 25:1-3.

ZIELHUIIS, R. L., 1978. Biological monitoring. Scandinavian Journal of Environmental Health, 4:1-18. 